\title{
Effects of Subtle Heterosexism on Gays, Lesbians, and Bisexuals
}

\author{
Shawn M. Burn, PhD \\ Kelly Kadlec, MA \\ Ryan Rexer, BS
}

\begin{abstract}
This study examined heterosexism that is not specifically targeted at LGB individuals, but may be experienced as antigay harassment, and may contribute to the stigma and stress they experience. LGB participants ( $N=175$, primarily Euro-American college students), read scenarios of heterosexuals saying or assuming things potentially offensive to gay men or lesbian women. For each scenario, they indicated the extent to which they would be offended and less open about their sexuality, and their perceptions of the behaviors as evidence of antigay prejudice. Not only did respondents find the scenarios to be offensive and indicative of prejudice, but perceived offensiveness was associated with a decreased likelihood of coming out. In comparison to gay men, lesbian women and bisexuals found the scenarios more offensive and more indicative of prejudice. Limitations of the current study and directions for future research are outlined.
\end{abstract}




\section{INTRODUCTION}

The United States has made strides toward becoming a less blatantly antigay society. However, heterosexism (prejudice against those that are not heterosexual) remains a problem. For instance, hate crimes that target individuals based on sexual orientation remain common, and subtle forms of sexual prejudice continue to remind lesbian, gay, and bisexual (LGB) persons of their lower status (Herek, 2000; Herek, Cogan, \& Gillis, 2002). Herek (1989) found that as many as $92 \%$ of lesbian women and gay men have been targets of antigay verbal abuse or threats. Similarly, D'Augelli and Rose (1990) found that nearly three-fourths of lesbian women and gay men respondents in a university community had experienced verbal abuse and that nearly all of them expected future harassment. In a more recent study, 94\% of LGB adults surveyed described being a victim of at least one hate crime based on sexual orientation (Herek et al., 2002).

Antigay harassment includes verbal or physical behavior that injures, interferes with, or intimidates lesbian women, gay men, and bisexual individuals. Anti-gay harassment also includes the indirect suggestion, conveyed through jokes and comments, that homosexuals and bisexuals are unwelcome and abnormal. The pejorative words young heterosexuals use to deride one another frequently include remarks specific to LGB persons (Burn, 2000; DiPlacido, 1998; Plummer, 2001; Thurlow, 2001). Burn (2000) found that heterosexual male college students in the United States frequently used antigay language (e.g., "faggot" and "queer" as insults). Further, it is currently fashionable for young American heterosexuals to use the word "gay" as an adjective when referring to a person, place, or thing, as being "stupid" or undesirable. In Britain, Thurlow (2001) found that heterosexist language is among the most frequently used pejorative terms, accompanying other categories such as racism, sexism, and phallocentrism. Heterosexist expressions and sentiments also are used in response to those who violate traditional gender-role behavior (Friend, 1998). Heterosexuals not conforming to 
traditional gender stereotypes are often labeled "gay," "dyke," "butch," or "fag," or are assumed to be gay or lesbian with the implication that this is highly undesirable and a label to be avoided.

Herek (1990) contends that not all of those who exhibit gay bias are strongly heterosexist. Plummer (2001) suggests that boys use homophobic language to point out their target's "lack of allegiance to the collective expectations of male peers" (p. 21) and not to intentionally display prejudice toward LGB persons. The prevalence of this language in our culture is partly due to the relative invisibility of LGB persons. Heterosexuals, deprived of seeing whom their comments ultimately harm, are not inclined to carefully monitor their colloquial speech (Thurlow, 2001). There also is evidence that heterosexuals have completely dissociated homophobic language from its relationship to sexual orientation (Thurlow, 2001). For instance, when heterosexuals in the United States use the word "gay" to call someone or something "stupid," they may not perceive the word "gay" as being associated with sexual orientation. In Burn's (2000) study, about half of the people who used heterosexist language were not strongly anti-homosexual. For these individuals, the behavior may serve a social expressive function, helping them to win social approval and acceptance in their social group (Burn, 2000).

Even if heterosexist language is not used to intentionally harm LGB persons, it may be experienced as antigay harassment and contribute to psychosocial stress. Experiences of negative treatment in society and resultant lack of self-acceptance culminate to produce abnormally high chronic stress for LGB persons (Meyer, 1995). Research finds a number of negative effects from the stress related to stigmatization based on sexual orientation (APA Division 44, 2000). This stress is linked to depression, higher suicide rates among LGB persons during young adulthood (D'Augelli, 1992; Rofes, 1983; Rotheram-Borus, Hunter, \& Rosario, 1994; Savin-Williams, 1994), high-risk sexual behaviors (Folkman, Chesney, Pollack, \& Phillips, 1992; RothermanBorus, Reid, Rosario, \& Kasen, 1995), eating disorders (Brown, 1986), school problems (Bendet, 1986), substance abuse, running away, and prostitution (APA Division 44, 2000). The experience of anti-gay harassment has been found to be more common among gay and bisexual male adolescents who had attempted suicide than among those who had not (Rotheram-Borus et al., 1994).

The looming expectation of social rejection and antigay harassment may explain why so many LGB persons feel compelled to keep their sexuality secret or even feign heterosexuality (Bourassa \& Shipton 
1991). Unfortunately, LGB persons who are unsure of how people will respond to their sexual orientation once they have come out sustain additional stress as they attempt to conceal it (Meyer, 1995). Moreover, this self-concealment is related to significant physical and psychological problems (D'Augelli \& Rose, 1990; Larson \& Chastain, 1990). In one study, almost half of the lesbian and gay undergraduates surveyed were "not at all comfortable" disclosing their sexual orientation and two-thirds occasionally feared for their personal safety (D'Augelli, 1989). In addition, $80 \%$ concealed their sexuality from roommates, $89 \%$ from other undergraduates, $65 \%$ from faculty, and $70 \%$ from job supervisors. Moreover, to avoid harassment, almost half made significant life changes, including presenting oneself as heterosexual and avoiding other lesbian women and gay men.

A heterosexist climate also may contribute to internal homophobia. Internal homophobia occurs when an LGB person incorporates societal antigay attitudes into her or his self-image and these attitudes interfere with the development of a positive LGB identity (D'Augelli, 1992; DiPlacido, 1998). Shame about one's sexual orientation leads to fear of discovery, lowered self-esteem, denial, discomfort with being gay, an aggressive posture against other LGB persons, and feelings of isolation (Morrow, 1996; Sears, 1997). Even subtle antigay harassment may be detrimental to young LGB persons who are in the process of coming out to themselves and to others for the first time. The coming out process is an especially vulnerable time, during which antigay hostility and bias can hamper healthy development of a LGB identity (Blumstein \& Schwartz, 1993). Delayed coming out due to lack of self-acceptance has been shown to result in lessened interest in emotional intimacy and lessened self-esteem (Harry \& DeVall, 1978).

The stress and difficulties associated with hiding one's sexual orientation are significant for individuals but there are larger, social effects as well. One of the most important factors in overcoming stereotypes is to obtain personal information about other group members. Research indicates that people will set aside their stereotypes and judge people on an individual basis when personal information is available to them (Hilton \& Fein, 1989; Lord et al., 1994) and when they are highly motivated to form an accurate impression of someone (Hilton \& Darley, 1991; Snyder, 1992). Likewise, research indicates that coming out is, perhaps, the most potent method of reducing antigay sentiments (Davis, 1992; Garnets \& Kimmel, 1993; Gonsiorek \& Weinrich, 1991; Klein, 1993). More positive attitudes toward homosexuals are associated with knowing at least one individual who is lesbian or gay (D'Augelli \& Rose, 
1990; Herek, 1984; Herek \& Glunt, 1993), presumably because contact with LGB people replaces inaccurate myths regarding with more accurate, positive truths (Bridgewater, 1997). Of course, most homosexuals are not very open about their sexual orientation, due to their beliefs that they are likely to face prejudice and discrimination. Indeed, research shows that being out puts people at higher risk of experiencing discrimination, rejection, and violence (Bradford, Ryan, \& Rothblum, 1994). This tendency for GLB persons to remain closeted means that the content of heterosexuals' stereotypes of homosexuals is not derived from experience but rather from heterosexual culture and what other heterosexuals teach about lesbian women and gay men.

The current study examined the effects of subtle heterosexist remarks and assumptions on LGB persons. The focus was not on direct insults or harassment of LGB persons. Instead, it was on things said or assumed by heterosexuals that may be indicative of heterosexism, but that are indirect in their effects. Calling an openly gay male the epithet "faggot" is direct antigay harassment. Heterosexual males derogatorily calling one another "faggot" is an instance of indirect antigay harassment in that nearby LGB persons who overhear the comment may experience discrimination vicariously. Burn (2000) found that non-prejudiced heterosexuals became motivated to change their behavior once they considered how LGB individuals might feel when hearing antigay language. Therefore, one of the purposes of the present study was to examine the assumption that such language contributes to the stigmatization experienced by LGB persons and reduces the likelihood of their coming out. Such information then could be used in educational campaigns to motivate the reduction of this type of behavior. The study hypotheses were as follows:

1. LGB people would be offended by the behaviors exhibited by heterosexuals in hypothetical scenarios.

2. The heterosexist behaviors, though not directed at LGB persons, would decrease likelihood of openness about sexual orientation.

3. Greater offensiveness would be associated with a decreased likelihood of coming out.

4. The heterosexuals in the scenarios would be perceived as prejudiced against gay men and against lesbian women.

There was also interest in comparing lesbian women, gay men, and bisexuals on the dependent variables, although no specific hypotheses 
were formulated. Would there be group differences in the perceived offensiveness of the scenarios or the perception of prejudice or the effect of the remarks on likelihood of coming out? Since the derogation of homosexuals is especially common in male culture, gay and bisexual males may be more accustomed to it and less affected by it. Would lesbian women and bisexual females be more affected by scenarios specific to their gender and gay and bisexual males by scenarios specific to theirs? Research on altruism indicates that similarity to the "victim" increases the empathic response (Houston, 1990). However, the group identity of non-heterosexual (homosexual or bisexual) may supercede these subgroup identifications such that similarity is perceived regardless of whether the remark is specific to lesbian women or gay men.

\section{METHOD}

\section{Participants}

Respondents were recruited from three sources. The first source $(n=$ 33) were participants in one of five Lesbian, Gay, Bisexual, and Transgender (LGBT)-type campus clubs (California Polytechnic State University, San Luis Obispo, University of California at Santa Barbara, University of Wisconsin, University of Wyoming, and University of Saskatchewan, Canada). A second source were attendees at a 2001 LGBT convention at UC Santa Barbara $(n=23)$. A third source were visitors to an Internet Web site $(n=119)$, on which was posted an electronic version of the survey. Respondents that clearly indicated gay, bisexual, or lesbian sexual orientation (175 of 217) were selected for study.

The sample $(n=175)$ was $48 \%(n=84)$ male and $52 \%(n=91)$ female. Sexual orientation was comprised of $75(42.9 \%)$ gay men, 67 (38\%) lesbian women, $24(13.7 \%)$ bisexual females, and $9(5.1 \%)$ bisexual males. Ethnic identification consisted of $79.4 \%(n=139)$ European-American/White, $6.3 \%(n=11)$ Latin-American/Hispanic, $4.6 \%$ $(n=8)$ Asian-American/Pacific Islander, $1.1 \%(n=2)$ African-American/Black, $1.1 \%(n=2)$ Middle-Eastern/Arab, and .6\% $(n=1)$ Native American. Two or more categories were checked by $5.7 \%(n=10)$ of participants and $1.1 \%(n=2)$ did not check any category. Most participants, 95.4\% $(n=167)$, had completed at least some college. Age ranged from 15-57 $(M=25.1, M d \mathrm{n}=22.0, S D=8.53)$, with two-thirds of participants between $18-25$ years old; two $(1.1 \%)$ respondents did not 
indicate age. A variety of college majors were represented across standard academic disciplines.

\section{Questionnaire}

After providing informed consent, the participants read 13 different one-sentence scenarios of heterosexuals saying or assuming things that might be offensive to gays or lesbian women (see Table 1). The items were developed according to what was believed to be representative scenarios of what LGB people frequently encounter. The scenarios were piloted on small groups of LGB people, who agreed on their content.

For each of the 13 scenarios, a 7-point Likert scale $(7=$ Strongly Agree, $1=$ Strongly Disagree) was used to indicate extent of agreement with each of the following four statements: "I would be offended." "I

TABLE 1. Subtle Heterosexism Scenarios

1. A heterosexual calls a man who isn't athletic a "fag."

2. A heterosexual assumes that two unmarried women who spend a lot of time together are lesbian women.

3. A man who pursues an occupation commonly pursued by females is assumed to be gay by a heterosexual.

4. A heterosexual assumes that women who are not traditionally feminine are lesbian women.

5. A heterosexual assumes two unmarried men who spend a lot of time together are gay.

6. A heterosexual calls an athletic female a "dyke."

7. Men who are not traditionally masculine are assumed to be gay by a heterosexual.

8. A heterosexual calls a woman who shuns makeup and has short hair "butch."

9. A heterosexual calls a man who is crying a "fag."

10. An unattractive female is assumed to be a lesbian by a heterosexual.

11. The term "gay" is used by a heterosexual to refer to someone who is perceived as stupid.

12. While watching a television show that features lesbian women or gays, the heterosexual viewers begin making a series of jokes about gays and lesbian women.

13. "Queer," "fag," "gay," or "dyke" is used when playfully trading insults amongst heterosexuals. 
would be less likely to be open about my sexual orientation." "I would assume that a heterosexual who says or does this is prejudiced against gay men." "I would assume that a heterosexual who says or does this is prejudiced against lesbian women." Pretesting indicated that having some positively scored and some negatively scored items was confusing given the number of responses required (52). Participants tended to respond as if all items were similarly keyed and expressed a preference for a more straightforward questionnaire without item reversals. This is consistent with some research finding that negations are cognitively burdensome and error-laden for respondents (Visser, Krosnick, \& Lavrakas, 2000).

Participants also responded to demographic questions regarding age, gender (female, male, or other), sexual orientation (gay/lesbian, bisexual, heterosexual), education (13 categories provided), college major (open-ended), hometown, and ethnicity ( 7 categories provided including, "other, please specify"). Respondents were instructed to check all that applied.

\section{Procedure}

The procedures for data administration and collection varied depending on the sub-sample and are described separately.

Campus Clubs. Leaders of five university LGBT-type student clubs within driving distance of the researchers were contacted by phone or e-mail to request participation in the study. It was explained that participation of members was voluntary, anonymous, and that a packet of surveys with administration instructions and a self-addressed stamped envelope could be sent, or that the researchers could attend a meeting and administer the survey. Two clubs agreed to participate by having two of the researchers (one female, one male) arrive at the beginning of a meeting and administer the surveys. The researchers reviewed the instructions and emphasized that participation was voluntary and anonymous. Participants placed their completed surveys in an unmarked envelope at the front of the room. The other three clubs requested a packet of surveys along with self-addressed stamped envelopes to return completed surveys.

Internet. A second set of LGBT-type club leaders were contacted via e-mail addresses obtained from gay Website directories. In the e-mail, the purpose of the project was explained and two options for member participation were provided. Club leaders could either receive a packet of surveys as described above, or refer their members to a Web page with 
an electronic version (coded in HTML) of the informed consent. Web participants provided informed consent by selecting the "I agree" hyperlink, directing them to the survey Web page. Results were returned electronically.

UCLGBTA Conference. Two of the researchers (one female, one male) attended the 2001 University of California, Lesbian, Gay, Bisexual, and Transgender Association (UCLGBTA) convention in southern/central California (Santa Barbara). The UCLGBTA is an annual convention with diverse educational, political, and relationship workshops pertinent to LGBT people. The two researchers set up a table at a general information area and staffed it for a total of six hours over the course of the weekend. Conference attendees that passed by the table were politely asked to complete the survey. They submitted the completed surveys to one of the researchers, who immediately placed them in an unmarked envelope.

\section{RESULTS}

Unless otherwise noted, alpha for all analyses was .05. Also, due to ordinal data, non-parametric statistics were used (Seigel \& Castellan, 1988). In the initial examination of the data, a Levene test for equality of variances did not reveal significant differences between the three sub-samples on their responses to dependent measures. Hence, due to small $n$ s in two of the sub-sample groups, they were combined for analyses $(n=175)$. The Levene test also did not reveal a significant difference between the variance of female bisexuals' responses $(n=24)$ and the variance of male bisexuals' $(n=9)$ responses. They were combined into a single bisexual group $(n=33)$ because their group $n$ s were too small to run analyses on them independently.

\section{Offensiveness of the Scenarios}

The offensiveness ratings were summed to create an offensiveness index with high internal reliability (Cronbach's $\alpha=.93$ ). The same procedure was used when computing Cronbach's alpha for each of the other three scales. The closer the rating was to 7, the more strongly the participant agreed that she or he would be offended. In general, participants agreed that they would be offended by the scenarios. The mean $(n=167)$ of the 13 offensiveness ratings was 5.8, $S D=1$.1. Hence, the hypothesis 
was supported that LGB persons are offended by the indirect antigay harassment exhibited by heterosexuals. For gay men $(n=70)$ the mean was 5.4, $S D=1.3$; for lesbian women $(n=65)$ it was $6.1, S D=.7$; and for bisexuals $(n=32)$ it was 6.0, $S D=1.0$. A Kruskall-Wallis test indicated that the offensiveness ratings differed significantly by group, $\chi^{2}(2, \mathrm{~N}=$ $167)=19.59, p<.001$. Mann-Whitney tests with a Bonferroni adjustment were used for this and all further pairwise comparisons $(\alpha=.017)$. The tests indicated that both lesbian women and bisexual individuals were significantly more offended than were gay men, $z=-4.01, p<.001$, and $z=-3.25, p<.001$, respectively. However, lesbian women and bisexual individuals did not significantly differ $(z=-.45, p=. \mathrm{ns})$.

\section{Effect of Remarks on Likelihood of Coming Out}

A summed index of likelihood of coming out ratings was created $(\alpha=$ .97). Participants' ratings $(n=164)$ indicated that hearing such remarks would not affect the likelihood that they would be open about their sexual orientation $(M=4.0, S D=1.8)$. Further, although the groups did not differ significantly $\chi^{2}(2, \mathrm{~N}=164)=3.6, p=. \mathrm{ns}$, there was a trend of higher ratings from lesbian women $(M=4.2, S D=1.8)$ and bisexual individuals $(M=4.2, S D=1.8)$ than from gay men $(M=3.7, S D=1.8)$. However, a post hoc Spearman's rho revealed that perceived offensiveness was associated with a decreased likelihood of coming out, $r_{\mathrm{s}}(164)=$ $.25, p<.001$, thus supporting the hypothesized relationship between likelihood of coming out and perceived offensiveness of the behaviors.

\section{Effect of Remarks on Perceived Prejudice Against Gay Men}

The summed ratings of how indicative of prejudice against gay men the scenarios were perceived to be yielded a Cronbach's alpha of .87 . Participants $(n=162)$ somewhat agreed that they would assume that a heterosexual who made the remarks is prejudiced against gay men $(M=$ $5.3, S D=.9$ ). Thus, the hypothesis that LGB people perceive heterosexuals who participate in these behaviors as prejudiced against gay men was supported. The three groups' ratings differed significantly, $\chi^{2}(2, \mathrm{~N}=$ $175)=12.21, p<.002$. Lesbian women's ratings $(M=5.6, S D=.7)$ were significantly higher than gay men's ratings $(M=5.1, S D=.96, z=$ $-3.42, p<.001)$, but did not differ from bisexuals' ratings $(M=5.4, S D=$ 
$.86, z=-.47, p=. n s)$. Bisexuals' ratings were not significantly greater than gay men's ratings $(z=-2.08, p=\mathrm{ns})$.

\section{Effect of Remarks on Perceived Prejudice Against Lesbian Women}

An index was created by summing the "I would assume that a heterosexual who says or does this is prejudiced against lesbians" ratings of the scenarios $(\alpha=.87)$. Participants' ratings $(N=162)$ suggested that they somewhat agreed they would assume heterosexuals who made the 13 remarks were prejudiced against lesbian women $(M=5.2, S D=.92)$. Hence, our hypothesis was supported that LGB people perceive heterosexuals who participate in these behaviors to be prejudiced against lesbian women. The groups' ratings differed significantly $\chi^{2}(2, \mathrm{~N}=162)=$ $17.23, p<.001$. The ratings of lesbian women $(M=5.5, S D=.8)$ were significantly higher than gay men's, $M=4.9, S D=.96, z=-3.9, p<$ .001 , but did not differ from bisexuals' $(M=5.4, S D=.9, z=-.153, p=$ $\mathrm{ns})$. Bisexuals' ratings also were significantly higher than gay men's ratings $(z=-2.86, p=.004)$.

\section{DISCUSSION}

As predicted, lesbian women, gay males and bisexual individuals found the 13 statements and assumptions generally offensive. Although LGB persons reported that for the most part the statements and assumptions would not influence the likelihood of their coming out, there was a small but significant association between the perceived offensiveness of the items and the likelihood of coming out. This is consistent with past research that shows LGB people are less likely to come out in hostile environments (D'Augelli, 1989; DiPlacido, 1998). The weakness of this effect may be because such remarks are only one factor affecting the complex decision of coming out. In terms of perceived prejudice, respondents somewhat agreed that heterosexuals who participated in the 13 scenarios were prejudiced against gay men and lesbian women. Past research demonstrates that heterosexuals often use this language without the objective of expressing sexual prejudice. This study points out the incongruity behind the intent and reception of such behavior. The study revealed some interesting group differences, in particular the finding that lesbian women and bisexual individuals found the items more offensive than gay males. This difference could reflect gay males' 
habituation to such behavior (i.e., it is a common part of male culture) and, thus, less likely to be perceived as offensive by men.

There are several methodological shortcomings of the study. One of these is that we lack proof that our measures are construct valid. Although face validity appears high, the measures were internally consistent, and judges deemed the scenarios to be representative of the types of remarks made by heterosexuals, we lack data on how common such scenarios are and whether we left out some important ones. Given the large number of items and that item reversals were not used, response sets may also have been a problem (as explained earlier, item reversals seemed to create more problems than they solved in this case). The sample size and its self-selected nature also inhibit the generalizability of the findings. Representative samples of this population are difficult to obtain.

The study also falls short in providing a thorough study of bisexuals. For instance, the number of bisexuals in our sample was not large enough to permit analysis by gender. In addition, we did not include scenarios depicting heterosexuals participating in biphobic behaviors. Primarily, this is a consequence of a lower incidence of behaviors indicative of biphobia. For instance, "fence-sitter" or "switch-hitter" is not used in quite the same manner as "fag" or "dyke." This is not to say that biphobia does not exist, but that it exists in a different manner than does antigay sentiment. In fact, bisexuals may be condemned by heterosexual individuals, gay men, and lesbian women (APA Division 44, 2000). Further research should specifically examine the stigmatization experiences of bisexuals.

We also did not measure "outness," and this may have affected our findings. For instance, those willing to respond to our survey may have been "more out." Therefore they may have had more of an opportunity to habituate to antigay sentiments, simply because they encounter them more frequently as more exposed targets for antigay harassment. Habituation may occur when LGB persons learn that, although harassment certainly does result in harm, the vast majority of it is not harmful enough to prevent them from expressing a core part of themselves-that is, their sexuality. An LGB person openly displaying his or her sexuality may do so precisely because he or she is no longer cowed by indirect heterosexist behaviors. Being out requires imperviousness to antigay sentiments. In summary, to the extent that the sample was indeed more out, this may explain why our scenarios of subtle heterosexism did not purportedly influence respondents' likelihood of being open about their sexual orientations. 
As mentioned, coming out is an extremely effective way to reduce antigay sentiment (D'Augelli \& Rose, 1990; Herek, 1984; Herek \& Glunt, 1993). Given the prevalence of antigay sentiment, it is surprising so many LGB people do come out. However, it should not be incumbent on them to come out in hostile environments in order to reduce prejudice. Heterosexuals ought to be responsible for the repercussions of the harm their language can cause and for establishing nurturing environments in which LGB people can feel safe in expressing their sexualities. Heterosexist individuals often do not realize the negative repercussions of their words and would modify their language if they were more cognizant of its effects (Burn, 2000). Results from the present study can be used as part of awareness campaigns and peer reminders that these types of remarks harm LGB persons. It should be emphasized that, although not overtly vicious, these types of remarks and assumptions are part of a heterosexist culture that contributes to the stigmatization of non-heterosexual orientations. Perhaps future researchers will design and test interventions based on these ideas.

\section{NOTES}

1. Respondents were disqualified for analyses where they had missing data.

2 . The thirteen item summaries by sexual orientation across the four scales may be requested from the senior author.

\section{REFERENCES}

APA Division 44/Committee on Lesbian, Gay, and Bisexual Concerns Joint Task Force on Guidelines for Psychotherapy with Lesbian, Gay, and Bisexual Clients. (2000). Guidelines for psychotherapy with lesbian, gay, and bisexual clients. American Psychologist, 55, 1440-1451.

Bendet, P. (1986, August-September). Hostile eyes: A report on homophobia on American campuses. Campus Voice, pp. 30-37.

Blumstein, P. W., \& Schwartz, P. (1993). Bisexuality: Some social psychology issues. In L. D. Garnets \& D. C. Kimmel (Eds.), Psychological perspectives on lesbian and gay male experiences (pp. 168-184). New York: Columbia University Press.

Bourassa, D. M., \& Shipton, B. (1991). Addressing lesbian and gay issues in residence hall environments. In N. J. Evans \& B. Shipton (Eds.), Beyond tolerance: Gays, lesbians, and bisexuals on campus (pp. 79-96). Alexandria, VA: American College Personnel Association. 
Bradford, J., Ryan, C., \& Rothblum, E. D. (1994). National lesbian health care survey: Implications for mental health care. Journal of Consulting and Clinical Psychology, 62, 228-242.

Bridgewater, D. (1997). Effective coming out: Self-disclosure strategies to reduce sexual identity bias. In J. T. Sears \& W. L. Williams (Eds.), Overcoming heterosexism and homophobia: Strategies that work (pp. 13-48). New York: Columbia University Press.

Brown, L. S. (1986). Confronting internalized oppression in sex therapy with lesbian women. Journal of Homosexuality, 12, 99-107.

Burn, S. M. (2000). Heterosexuals' use of "fag" and "queer" to deride one another: A contributor to heterosexism and stigma. Journal of Homosexuality, 40, 1-11.

D’Augelli, A. R. (1989). Lesbians' and gay men's experiences of discrimination and harassment in a university community. American Journal of Community Psychology, 17, 317-321.

D'Augelli, A. R. (1992). Lesbian and gay male undergraduates' experiences of harassment and fear on campus. Journal of Interpersonal Violence, 7, 383-395.

D’Augelli, A. R., \& Rose, M. L. (1990). Homophobia in a university community: Attitudes and experiences of heterosexual freshmen. Journal of College Student Development, 31, 484-491.

Davis, P. (1992). The role of disclosure in coming out among gay men. In K. Plummer (Ed.), Modern homosexualities: Fragments of lesbian and gay experience (pp. 7586). New York: Routledge.

DiPlacido, J. (1998). Minority stress among lesbian women, gay men, and bisexuals: A consequence of heterosexism, homophobia, and stigmatization. In G. Herek (Ed.), Stigma and sexual orientation: Understanding prejudice against lesbians, gaymen, and bisexuals (pp. 138-159). Thousand Oaks, CA: Sage.

Folkman, S., Chesney, M. A., Pollack, L., \& Phillips, C. (1992). Stress, control, coping and depressive mood in human immunodeficiency virus-positive and negative gay men in San Francisco. Journal of Nervous and Mental Disease, 181, 409-416.

Friend, R. A. (1998). Heterosexism, homophobia, and the culture of schooling. In S. Books, (Ed.), Invisible children in the society and its schools (pp. 137-166). Mahwah, New Jersey: Lawrence Erlbaum Associates.

Garnets, L. D., \& Kimmel, D. C. (1993). Psychological perspectives on lesbian and gay male experiences. New York: Columbia University Press.

Gonsiorek, J. C., \& Weinrich, J. D. (1991). Homosexuality: Research implications for public policy. Newbury Park, CA: Sage.

Harry, J., \& DeVall, W. B. (1978). The social organization of gay men. New York: Prager.

Herek, G. M. (2000). The psychology of sexual prejudice. Current Directions in Psychological Science, 9, 19-22.

Herek, G. M. (1984). Beyond "homophobia": A social psychological perspective on attitudes toward lesbians and gay men. In J. P. DeCecco (Ed.), Homphobia in American society: Bashers, batterers, and bigots (pp. 1-21). New York: Harrington Park Press.

Herek, G. M. (1989). Hate crimes against lesbians and gay men: Issues for research and policy. American Psychologist, 44, 948-955. 
Herek, G. M., Cogan, S. C., \& Gillis, J. R. (2002). Victim experiences of hate crimes based on sexual orientation. Journal of Social Issues, 58, 319-399.

Herek, G. M., \& Glunt, E. K. (1993). Interpersonal contact and heterosexuals' attitudes toward gay men: Results from a national survey. Journal of Sex Research, 30, 239-244.

Hilton, J. L., \& Darley, J. M. (1991). The effects of interaction goals on person perception. Advances in Experimental Social Psychology, 24, 235-267.

Hilton, J. L., \& Fein, S. (1989). The role of typical diagnosticity in stereotype-based judgments. Journal of Personality and Social Psychology, 57, 201-211.

Houston, D. A. (1990). Empathy and the self: Cognitive and emotional influences on the evaluation of negative affect in others. Journal of Personality and Social Psychology, 59, 859-868.

Klein, F. (1993). The bisexual option. New York: Harrington Park.

Larson, D. G., \& Chastain, R. L. (1990). Self-concealment: Conceptualization, measurement, and health implications. Journal of Social and Clinical Psychology, 9, 439-455.

Lord, C. G., Desforges, D. M., Fein, S., Pugh, M., \& Lepper, M. R. (1994). Typicality effects in attitudes toward social policies: A concept-mapping approach. Journal of Personality and Social Psychology, 66, 658-673.

Meyer, I. H. (1995). Minority stress and mental health in gay men. Journal of Health and Social Behavior, 7, 9-25.

Morrow, D. F. (1996). Coming-out issues for adult lesbian women: A group intervention. Social Work, 41, 647-656.

Plummer, D. C., (2001). The quest for modern manhood: Masculine stereotypes, peer culture and the social significance of homophobia. Journal of Adolescence, 24, 15-23.

Rofes, E. E., (1983). I thought people like that killed themselves: Lesbians, gay men and suicide. San Francisco: Grey Fox.

Rotheram-Borus, M., Hunter, J., \& Rosario, M. (1994). Suicidal behavior and gay-related stress among gay and bisexual male adolescents. Journal of Adolescent Research, 9, 498-508.

Rotheram-Borus, M. J., Reid, H., Rosario, M., \& Kasen, S. (1995). Determinants of safer sex patterns among gay/bisexual male adolescents. Journal of Adolescence, 18, 3-15.

Savin-Williams, R. C. (1994). Verbal and physical abuse as stressors in the lives of lesbian, gay male, and bisexual youths: Associations with school problems, running away, substance abuse, prostitution, and suicide. Journal of Consulting and Clinical Psychology, 62, 261-269.

Sears, J. T. (1997). Thinking critically/intervening effectively about heterosexism and homophobia: A twenty-five-year research retrospective. In J. T. Sears, \& W. L. Williams (Eds.), Overcoming heterosexism and homophobia: Strategies that work (pp. 13-48). New York: Columbia University Press.

Seigel, S., \& Castellan, N. J. Jr. (1988). Non-parametric statistics for the behavioral sciences (2nd ed.). New York: McGraw-Hill. 
Snyder, M. (1992). Motivational foundations of behavioral confirmation. In M. P. Zanna (Ed.), Advances in experimental social psychology (Vol. 25, pp. 67-114). San Diego: Academic Press.

Thurlow, C., (2001). Naming the "outsider within": Homophobic pejoratives and the verbal abuse of lesbian, gay and bisexual high-school pupils. Journal of Adolescence, 24, 25-38

Visser, P. S., Krosnick, J. A., \& Lavrakas, P. J. (2000). Survey research. In H. T. Reis \& C. M. Judd (Eds.), Handbook of research methods in social and personality psychology (pp. 223-252). Cambridge, UK: Cambridge University Press. 